\title{
GAS CIRCUNDANTE A UN HALO DE MATERIA OBSCURA
}

\author{
Gas around a dark matter halo
}

EPISTEMUS

ISSN: 2007-8196 (electrónico) ISSN: 2007-4530 (impresa)

\section{Guillermo Arreaga García ${ }^{1}$ Silvio Oreste Topa ${ }^{2}$}

Recibido: 20 de mayo de 2016,

Aceptado: 30 de octubre de 2016

Autor de Correspondencia:

Dr. Guillermo Arreaga García

Correo: guillermo.arreagag@gmail.com

\section{Resumen}

Haciendo uso de la técnica SPH (Smooth Particle Hydrodynamic) hidrodinámica con partículas suavizadas, realizamos la evolución dinámica de una región representativa del Universo observable. Comparamos los resultados de un modelo cosmológico representado por dos simulaciones, a saber: la de base, en la cual la proporción del número de partículas de materia obscura y de gas es de uno a uno; y la simulación modificada, en la cual aumentamos el número de partículas de gas con la técnica conocida como Particle splitting, mientras dejamos el número de partículas de materia obscura sin cambio como una primera aproximación. El propósito de tales simulaciones es comparar las propiedades de aglomeración del gas, tanto del original como del adicional, en torno a un halo de materia obscura escogido. En este trabajo, nos conformamos con hacer una comparación cualitativa mediante gráficas, en las cuales mostramos como resultado principal que el gas adicional sigue un patrón filamental de formación de cúmulos densos dirigidos hacia el halo de materia obscura escogido.

Palabras clave: Hidrodinámica, técnicas computacionales, estructura de largo alcance del universo, cosmología.

\begin{abstract}
Making use of the SPH (Smooth Particle Hydrodynamic) technique, we carry out the dynamical evolution of a representative region of the observable Universe. We compare the outcomes of a cosmological model represented by two simulations, as follows: the base simulation, in which the proportion in the number of dark matter particles and gas particles is one to one; and the modified simulation, in which we increase the number of gas particles according the Particle splitting technique, while we leave the number of dark matter particles unchanged as a first approximation. The purpose of such simulations is to compare the agglomeration properties of the gas, both the original and the additional, around a chosen dark matter halo. In this paper, we settle for a qualitative comparison by means of plots, in which we show as the main result that the added gas follows a filament pattern of dense clumps formation toward the chosen dark matter halo.
\end{abstract}

Key words: Hydrodynamics, mathematical procedures and computer techniques, large scale structure of the universe, cosmology.

1 Departamento de Investigación en Física, Universidad de Sonora/Correo: garreaga@cifus.uson.mx

2 Eesa N 1 Tornquist, Provincia De Buenos Aires,Argentina/Correo: silviotopa@gmail.com 


\section{INTRODUCCIÓN}

El modelo cosmológico teórico más exitoso hasta el hoy día, está basado en la teoría de la relatividad general y en el Principio Cosmológico, que postula que toda región del Universo es estadísticamente igual a cualquiera otra, siempre que las dimensiones espaciales de tales regiones sean muy grandes [1]. Tales dimensiones espaciales son por lo menos del orden de $100 \mathrm{Mpc}$ (megapársecs: Un pársec es una unidad de distancia usada en astrofísica equivalente a $3.0857 \times 10^{16} \mathrm{~m}$ ). El problema cosmológico de interés físico hoy día, consiste pues en modelar (y eventualmente entender) la formación de agregados de materia en escalas de longitud mucho menores: por ejemplo, las galaxias y los grupos de galaxias visibles desde telescopios terrestres, son del orden de $1 \mathrm{Mpc}$, como el caso del Grupo Local de Galaxias; las nubes moleculares a partir de las cuales se forman las estrellas, son visibles en escalas de menos de un pársec o hasta decenas de pársecs. Cabe mencionar que en estas escalas galácticas y/o estelares, las diferentes regiones del Universo se ven radicalmente diferentes, pues el Principio Cosmológico ya no es aplicable [2].

En términos teóricos, esto significa que la física de un modelo cosmológico se obtiene de resolver las ecuaciones de Einstein aplicadas a una región grande del Universo, en la cual el Principio Cosmológico sea aún aplicable, de tal forma que dicha región se pueda modelar mediante un espacio-tiempo homogéneo e isotrópico. De hecho, las observaciones realizadas recientemente de la radiación de fondo (Cosmic Microwave Background) revelan que el Universo, después de una evolución temporal de apenas 400 mil años, era aún muy homogéneo, de tal forma que diferentes regiones compartían ya los mismos valores promedio de densidad de masa y temperatura [3]. Estas observaciones son una de las justificaciones más importantes del Principio Cosmológico. Ahora bien, una consecuencia muy importante de las ecuaciones de la relatividad general es que muestran que este tipo de espacio-tiempo homogéneo son dinámicos: en general se expanden.

Un ingrediente adicional muy importante para especificar completamente un modelo cosmológico es el contenido global de materia. Es común usar las cantidades adimensionales $\Omega_{m}$ y $\Omega_{l}$ para referirse al contenido de materia total (obscura y bariónica) y energía obscura, respectivamente. El contenido de materia más familiar para nosotros, porque todo en el planeta Tierra está hecho de este tipo de materia, se especifica por $\Omega_{b}$ y se le conoce como materia bariónica. Un elemento dinámico adicional que también permite definir el tipo de modelo cosmológico en consideración es la velocidad de expansión del Universo, que se especifica por la constante de Hubble, que se mide en $\mathrm{km}$ por segundo por Mpc, esto es $H$; o bien mediante un número adimensional denotado por $h$ y que es tal que $H=h \times 100 \mathrm{~km} \mathrm{seg}^{-1} \mathrm{Mpc}^{-1}$. En la siguiente seccion se van a especificar los valores usados en este articulo para caracterizar el contenido de materia y expansion del Universo.

En términos computacionales, una simulación cosmológica se lleva a cabo en una caja o volumen de

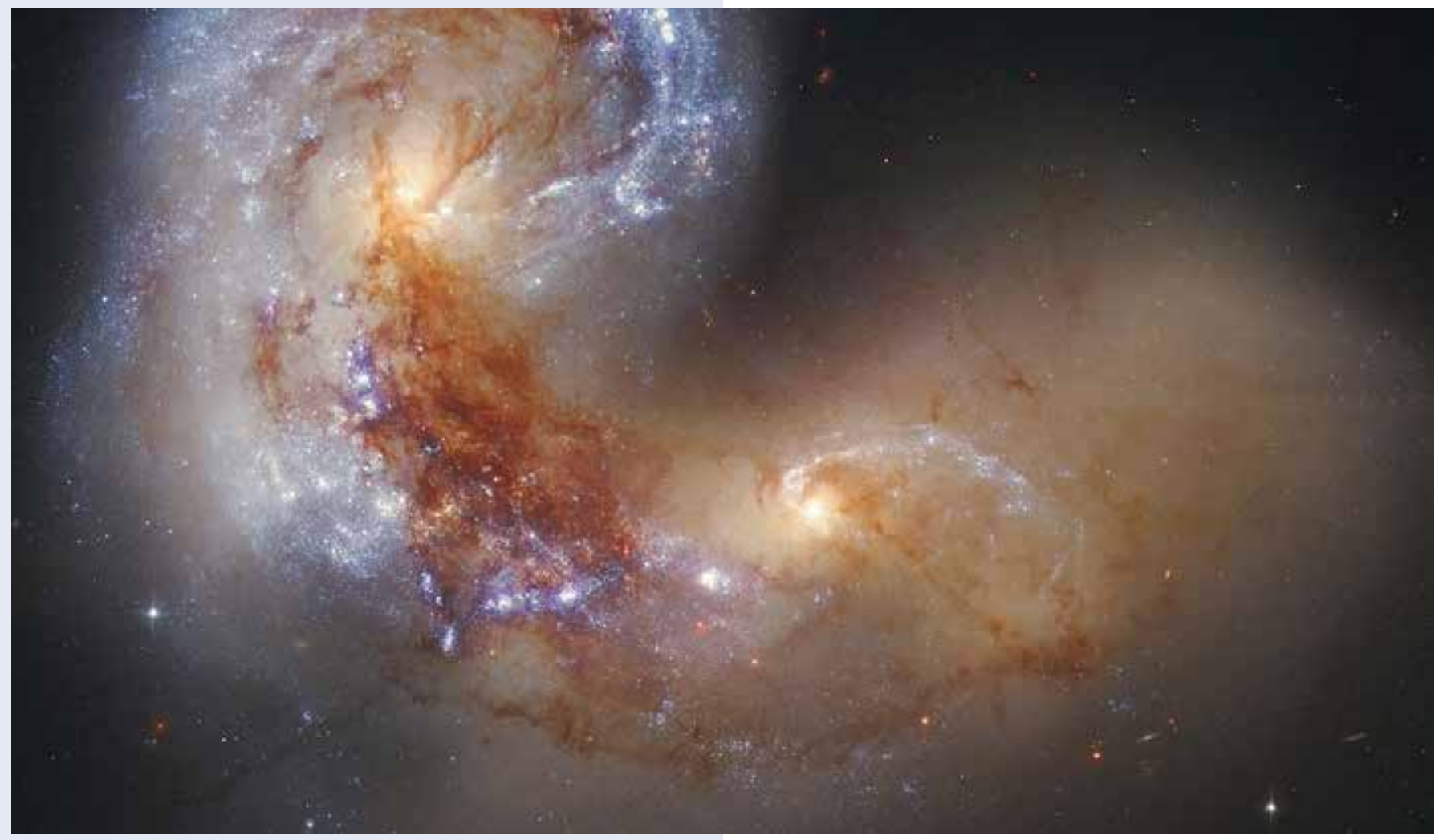


simulación, y la evolución se inicia en las etapas de alta homogeneidad, cuando el Principio Cosmológico es aún aplicable; se sigue la evolución temporal de esta región (delimitada por la caja de simulación) durante millones de años, de tal forma que pequeñas perturbaciones de densidad, plantadas a mano por el simulador, logran crecer hasta convertirse en las galaxias y estrellas que se observan hoy día a simple vista usando telescopios terrestres [4].

El desarrollo creciente de la capacidad de cómputo instalada y de fácil acceso en las universidades públicas; así como la propagación de la filosofía de compartir públicamente los códigos en la WEB 2, que se hace cada vez más popular y frecuente en el ámbito de la astrofísica, nos permiten hoy día estudiar el problema científico de la evolución del Universo en escalas cosmológicas, mediante simulaciones computacionales creadas usando únicamente códigos abiertos y libres. Este problema científico tiene importantes repercusiones, no sólo en el ámbito académico, sino también en el ámbito tecnológico; de hecho, varios de los grupos de cosmología computacional alrededor del mundo, promueven tanto el desarrollo de técnicas innovadoras de visualización como de nuevos y mejores algoritmos de cálculo [5].

Desde los años 70 se inició el área de simulaciones cosmológicas; de la mano con el avance en el hardware de los computadores, la resolución de las simulaciones (la resolución numérica es el producto del número de partículas usadas en una simulación por el rango espacial dinámico), ha crecido vertiginosamente en unos cuantos años: por ejemplo, en el año 2000, la simulación Millennium realizada principalmente por una colaboración entre Inglaterra y Alemania, requirió de un mes con toda la capacidad de cálculo del Centro de SuperCómputo del Instituto Max Planck en Garching, para evolucionar 10 mil millones de partículas de materia obscura usando la técnica SPH [6]. Más recientemente, la simulación Illustris, incluyó tanto materia obscura como gas, usando la técnica SPH, con un número de elementos dados por $1,820^{3}$, es decir, 6028 568000 partículas en total; adicionalmente, en el código de evolución también se incluyeron más procesos físicos relevantes, como el enfriamiento y la foto-ionización del gas, la formación estelar y la retro-alimentación dinámica estelar (feedback) [7]. Ambas simulaciones, Millennium e Illustris usaron variantes del código público GADGET, el cual será descrito brevemente en la siguiente sección, pues es el código que usamos para la realización de este trabajo.

\section{MÉTODO}

Consideremos una pequeña parte del Universo observable delimitado por una caja cúbica cuyo lado mide $L=105 M p c$. Consideremos que el contenido inicial de materia está caracterizado por $\Omega_{m}=0.2726$ y el contenido de energía obscura por $\Omega_{l}=0.7274$. La suma de estas cantidades $\Omega_{m}+\Omega_{l}=1.0$, que corresponde a un modelo de Universo plano en expansión dada por la constante de Hubble $H_{0}=100 \mathrm{~km} \mathrm{seg}^{-1} \mathrm{Mpc}^{-1}$ y el factor de indeterminación $h$, está dado por $h=0.704$. Estos valores escogidos para el contenido de materia son los más precisos que se hayan determinado usando las observaciones más recientes de la colaboración Planck 2013 [8]. La densidad media de masa en esta región del Universo por simular está dada entonces por $\rho=2.61 \times 10^{-30} \mathrm{gr} / \mathrm{cm}^{3}$.

Aunque sabemos que la Edad del Universo es de aproximadamente 13,800 millones de años, en este trabajo vamos a considerar que el estado inicial de la simulación corresponde a un Universo de 7.6 millones de edad, sólo por hacer menos costoso el proceso de simulación computacional. Los astrofísicos y cosmólogos acostumbran referirse al tiempo de evolución del Universo en términos del factor de escala del Universo, denotado por $a(t)$, en el cual este tiempo inicial correspondería $a\left(t_{i}\right)=0.007812$, mientras que el universo en el estado actual correspondería a un valor de $a\left(t_{\text {actual }}\right)=1$; o bien, a un redshift inicial de $z=127$, mientras que el universo actual correspondería a un redshift de $z=0$.

Hasta el día de hoy, en las áreas de la astrofísica y la cosmología numéricas, existen principalmente dos escuelas de simulación, de acuerdo con la técnica computacional usada para seguir la evolución de la materia, a saber: de malla adaptable o de partículas. En este trabajo, nos limitamos a explorar solo algunos programas públicos desarrollados usando la técnica de partículas: en particular, con la técnica SPH (Smooth Particle Hydrodynamics), en la cual las partículas representan al fluido de tal forma que al hacer el seguimiento temporal de las partículas nos permite simular la evolución temporal del fluido [5].

Así, llevamos a cabo la evolución temporal usando el código público GADGET-2, escrito en Ansi C y liberado por Volker Springel [9]. El GADGET-2 nos permite obtener la solucion de las ecuaciones de Euler-Navier-Stokes de la hidrodinámica clásicas (es decir, sin incluir ningún efecto relativista, con excepción de la expansión) escritas en términos de la técnica SPH sobre un número finito de partículas. Así, cada partícula es afectada por la fuerza gravitacional de las demás partículas y por las fuerzas debidas a los gradientes de presión y viscosidad. Cabe señalar que todas las fuerzas se suavizan con un kernel tipo spline. Usamos la ecuación de estado del gas ideal para caracterizar la termodinámica del gas.

El GADGET-2 se basa en el método del árbol (ParticleMesh) para el cálculo de las fuerzas gravitacionales entre partículase incorporalas siguientes características estándar: (i) cada partícula $i$ tiene su propia longitud de suavizado $h_{i}$; (ii) a las partículas también se les permite tener longitudes de suavizamiento gravitacional individuales $\varepsilon_{i}$, cuyos valores se ajustan de tal manera que en cada paso de tiempo $\varepsilon_{i} \times h_{i}$ es del orden de la unidad. GADGET-2 fija el valor de $\varepsilon_{i}$ para cada paso de tiempo utilizando el valor mínimo de la longitud de suavizado de todas las partículas, es decir, si $h_{\{\min \}}=\min \left(h_{i}\right)$ para $i=1,2 \ldots \mathbb{N}$, entonces $\varepsilon_{i}=h_{\{\min \}}$ para , entonces . El código GADGET-2 tiene una implementación 
de viscosidad artificial del tipo Monaghan-Balsara [10], [11], que consiste en agregar un término a la ecuación del momento según se establece en la segunda ley de Newton, de tal forma que esta fuerza de viscosidad permite simular la disipación de energía cinética en calor de fricción; esta fuerza se regula estableciendo los parámetros $\alpha_{v}=0.75$ y $\beta_{v}=\frac{3}{2} \alpha_{v}$, véase la ecuación (14) en [9]. Nosotros aquí fijamos el factor de Courant al valor 0.1.

Para generar un conjunto de perturbaciones de densidad consistente con la teoría cosmológica que sean plausibles de ser usadas como condiciones iníciales en nuestras simulaciones, usamos el programa público $\mathrm{N}$-GenlC escrito y liberado también por Volker Springel [12], el cual nos permite desplazar a las partículas inicialmente colocadas en el centro de una malla uniforme, de tal forma que el espectro de potencia teórico $P(k)$ en la representación de Fourier, con un número de onda definido por $k=\frac{2 \pi}{\lambda}$, tiene las características genéricas mostradas en la figura 1. La malla contiene 1,024 elementos de partición, por lo cual el rango de números de onda mínimo y máximo están dados por $k_{\min }=8.37 \times 10^{-2} \mathrm{~h} / \mathrm{Mpc}$ y por $k_{\text {max }}=8.57 \times 10^{1} \mathrm{~h} / \mathrm{Mpc}$.

En la simulación base de este trabajo, el número de partículas de materia obscura (DM) y gas (G) están dadas por $N_{D M}=2,097,152$ y por $N_{G}=2,097,152$, respectivamente. En términos de la masa del sol $M_{\odot}=1.99 \times 10^{33} \mathrm{gr}$, la masa de una partícula de materia obscura es de $1.26 \times 10^{10} M_{\odot}$ mientras que la masa de una partícula de gas es de $2.54 \times 10^{9} M_{\odot}$. Por lo tanto, la masa total de la simulación es $3.17 \times 10^{16} M_{\odot}$ y la fracción de masa de la materia obscura es 0.832 mientras que la fracción de masa del gas es de 0.167 .

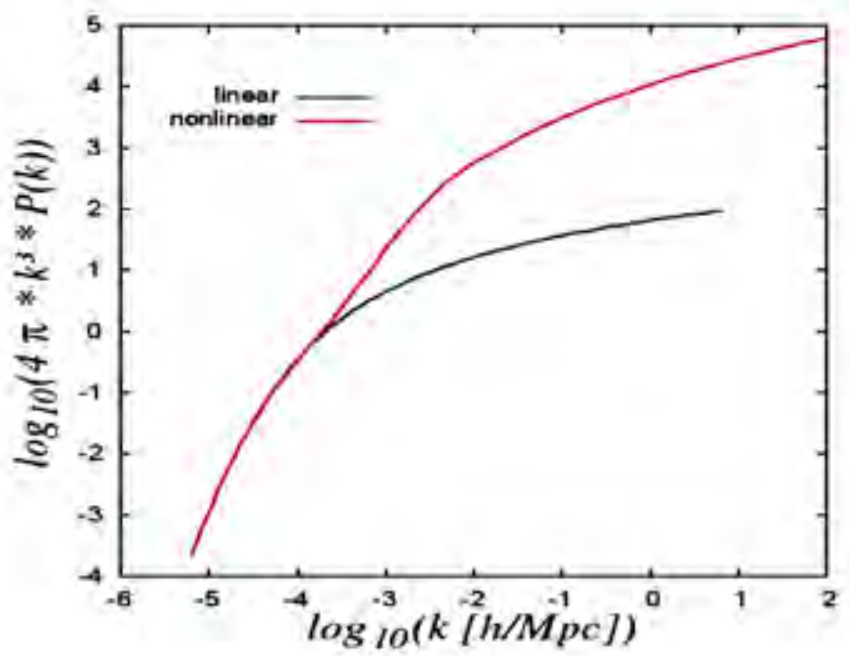

Figura 1. Espectro de potencia teórico.

Cabe señalar que el programa $\mathrm{N}-$ GenIC genera el conjunto inicial de partículas por parejas, esto es, por cada partícula de materia obscura hay una de gas. Una manera de mejorar el seguimiento de un componente particular de una simulación general es aumentando el número

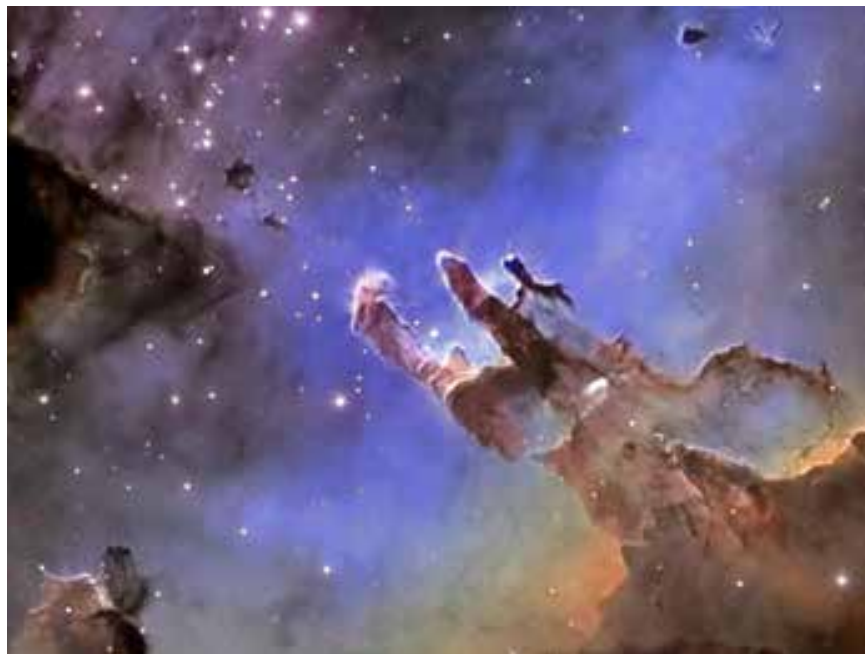

de partículas que se usa para representarlo en la caja de simulación. Por esta razón, una primera modificación de la simulación base que nos interesa probar en este trabajo es poner más partículas de gas en el volumen de simulación, de tal manera que por cada partícula de gas pondremos en su lugar $\mathrm{Ng}$ partículas de gas adicionales, pero con la masa inicial ajustada de tal forma que no cambien los parámetros globales de masa de la simulación.

De hecho, fue en simulaciones numéricas del colapso gravitacional, que esta técnica de multiplicación de partículas (Particle splitting) se introdujo, entre otros autores, por Kitsionas [13]. En cosmología numérica, variantes de esta técnica han sido usadas desde hace muchos años con el propósito de aumentar la resolución de una simulación que en general sólo incluían partículas de materia obscura, ver ejemplo en [14].

En la simulación modificada dejamos sin cambio a las partículas de materia obscura, por dos razones básicamente. La primera es que hay muchos trabajos de investigación en los que se demuestran las propiedades de convergencia en la evolución de la materia obscura, cuando se aumenta la resolución mediante un mayor número de partículas [14]. En esas primeras etapas, casi no se consideraba gas porque el interés principal estaba en escalas grandes y en la formación de los halos de materia obscura; tampoco se contaba con la capacidad de cómputo para considerar simulaciones con dos componentes de materia. La segunda razón es que queremos estudiar las propiedades de aglomeración del gas en el seno de un conjunto fijo de halos de materia obscura. Cabe mencionar que en las simulaciones hidrodinámicas del colapso gravitacional de nubes que se realizan para simular la formación estelar, cuando se aumenta el número de partículas, es posible a veces observar la aparición de efectos que corrigen a las simulaciones de menor resolución. De esta forma, los números totales de partículas para esta simulación modificada son ahora $N_{D M}=2,097,152$ y $N_{G}=23,068,672$, respectivamente. La masa de una nueva partícula de gas en esta simulación es de $2.54 \times 10^{8} M_{\odot}$. 


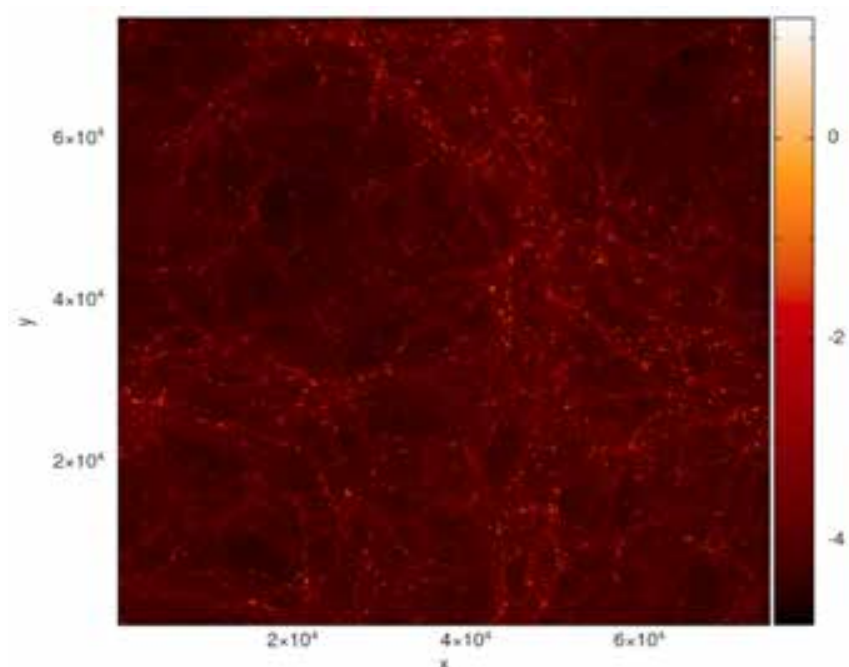

Figura 2. Corte transversal, paralelo al plano $\mathrm{XY}$, con la distribución de partículas de materia obscura y de gas al

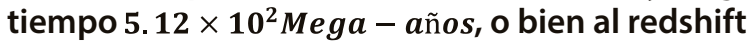
$z=0.91$. La barra lateral indica log de la densidad (en unidades del código).

\section{RESULTADOS}

La evolución temporal de la simulación de base requiere poco más de 500 horas de CPU, corriendo en paralelo en 20 procesadores INTEL Xeon a 3.2 Ghz del clúster científico Mezquite, propiedad del Área de Cómputo de Alto Rendimiento de la Universidad de Sonora. La evolución temporal de la simulación modificada es obviamente mucho más lenta que en el caso de la simulación base. Puesto que este tipo de simulaciones cosmológicas demandan un alto costo computacional, no es posible realizar más corridas usando sólo el equipo disponible en nuestra institución. Por esta razón, los objetivos de este trabajo se limitan a ser meramente ilustrativos de esta área del conocimiento en lugar de ser exhaustivos. Por ejemplo, no hemos realizado aquí un estudio de consistencia de las simulaciones con respecto a parámetros importantes de las corridas, que fueron fijados siguiendo la manera usual, que se sabe funciona, aprovechando nuestra experiencia haciendo simulaciones numéricas en otras áreas afines [15].

Con el propósito de visualizar el resultado de la evolución temporal, usamos el código público SPLASH, escrito y liberado por Daniel Price [16], de tal forma que un corte transversal de la caja para las simulaciones de base y modificada se muestra en las figuras 2 y 3 , respectivamente. Es interesante destacar la estructura fibrada que se observa en estas figuras. Las regiones más brillantes representan materia con estados de densidad mayor, de acuerdo con la escala de colores que se muestra en la parte derecha de cada figura. Se debe notar que en la figura 2 hay más regiones obscuras que en la figura 3: esto se debe al gas adicional que hemos proporcionado en la simulación modificada.

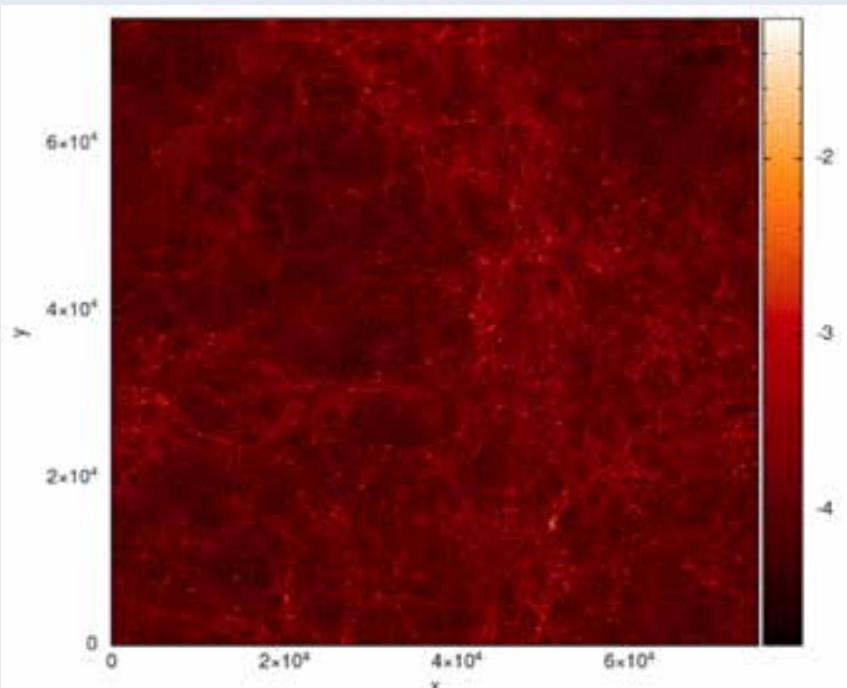

Figura 3. Corte transversal paralelo al plano $X Y$ después

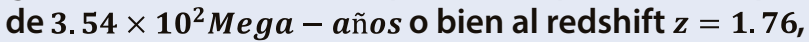
con la distribución de materia encontrada para la simulación modificada.

Una manera de comparar los resultados de las simulaciones, es aplicando el programa FoF_Special escrito y liberado por Volker Springel [17]. Este programa permite determinar las partículas que se agrupan formando cúmulos más densos mediante la técnica de Friends of Friends. Este algoritmo FoF_Special usa una longitud de ligamiento, que en este trabajo se fija como un factor 0.1 de la separacion minima promedio de las particulas. De tal forma que una partícula queda ligada a otras, siempre que éstas estén a menor distancia de la primera que esa longitud de ligamiento. Los cúmulos de partículas ligadas pueden representar tanto halos de materia obscura como nubes de gas.

Para la simulación de base, usando una longitud de ligamiento de $110,251 \mathrm{kpc} / \mathrm{h}$, fueron encontrados 1'362,988 grupos, pero sólo 3,100 grupos tuvieron más de 32 partículas de materia obscura; el grupo mayor localizado contiene 24,044 partículas, de las cuales 12,995 son de materia obscura y 11,049 de gas. Para la simulación modificada, usando una longitud de ligamiento de $109,643 \mathrm{kpc} / \mathrm{h}$, encontramos 1'582,469 grupos de partículas, aunque sólo 2,433 grupos tuvieron más de 32 partículas de materia obscura. El grupo mayor localizado contiene 66,801 partículas, de las cuales 5,749 son de materia obscura y 61,052 son de gas.

Se debe notar que las longitudes de ligamiento son prácticamente del mismo orden en ambas simulaciones. En el grupo mayor de la simulación de base, la proporción de partículas obscuras y de gas ligadas es muy similar: $54 \%$ y $45 \%$, respectivamente. En la simulación modificada estas proporciones cambian drásticamente: $9 \%$ de las partículas ligadas son de materia obscura mientras que $91 \%$ son de gas. 


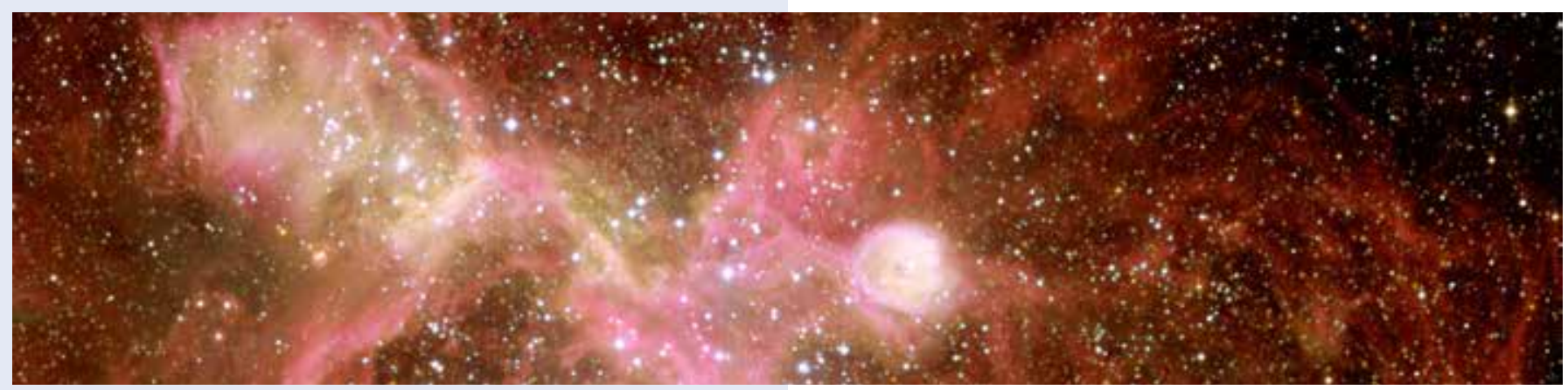

Una representación gráfica de los resultados obtenidos se muestra en las figuras 4 y 5 , en las cuales se debe notar que los grupos se distribuyen en todo el volumen de simulación, aunque si se alcanza a notar que los grupos siguen estructuras longitudinales, de acuerdo con lo observado previamente en las figuras 2 y 3 . Comparando estas figuras 4 y 5 , se puede ver que el gas adicional de la simulación modificada se distribuye en torno a los halos de materia obscura encontrados previamente en la simulación de base. Esta es una buena señal, pues indica que hay consistencia en las dos simulaciones de este trabajo.

Figura 4. Corte transversal paralelo al plano XY para mostrar los grupos localizados para la simulación cosmológica de base: en azul se muestran los grupos de materia obscura y en rojo los grupos de gas.

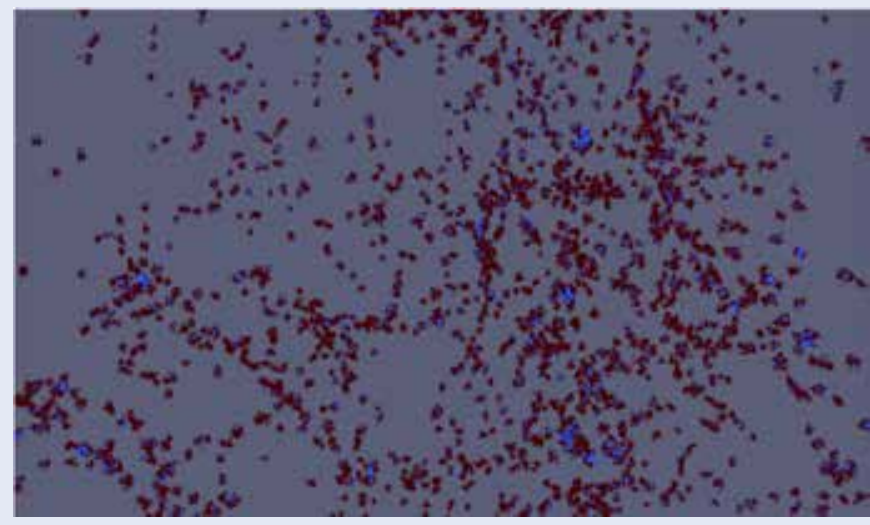

Figura 5. Corte transversal paralelo al plano $\mathrm{XY}$ para mostrar los grupos localizados para la simulación cosmológica modificada: en azul se muestran los grupos de materia obscura y en rojo los grupos de gas.

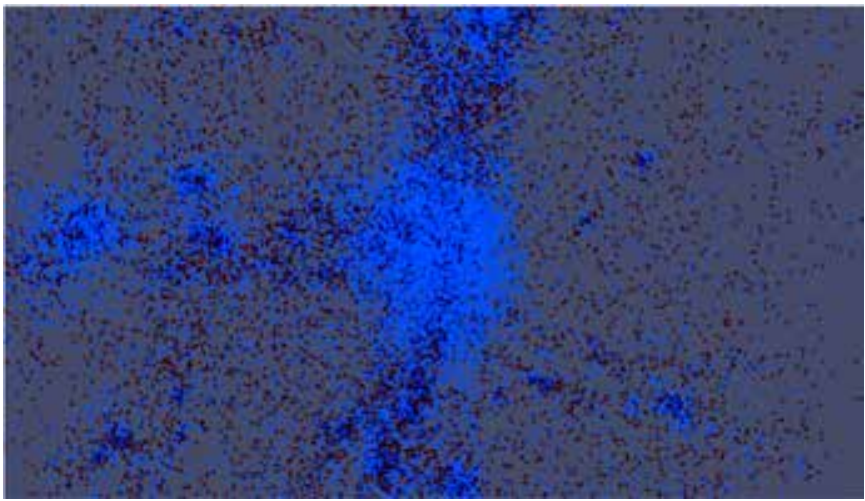

Figura 6. Corte transversal paralelo al plano XY para mostrar la región del halo mayor encontrado en la simulación base: las partículas de materia obscura se indican en color azul y las de gas en color rojo.

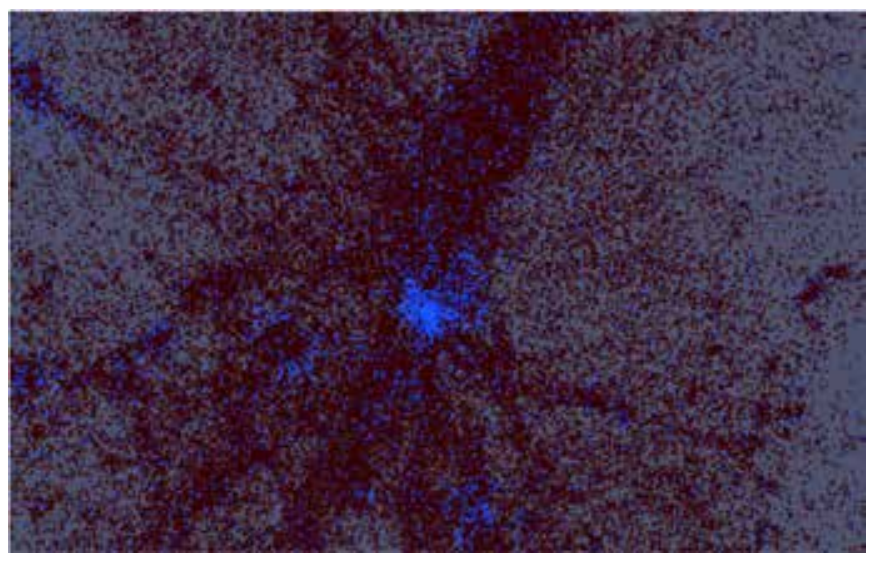

Figura 7. Corte transversal paralelo al plano XY para mostrar la región del halo mayor encontrado en la simulación modificada: las partículas de materia obscura se indican en color azul y las de gas en color rojo.

Conviene hacer ahora un acercamiento al grupo mayor detectado por el programa FoF_Special, pues este grupo constituye el halo más grande tanto en las simulaciones de base como modificada, que se muestran en las figuras 6 y 7 , respectivamente. Esta comparación visual nos permite detectar ligeros cambios en la estructura de los halos de materia obscura y en el gas circundante, lo cual es otra buena señal pues demuestra consistencia entre las dos simulaciones cosmológicas consideradas en este trabajo. 
Se debe mencionar que en la construcción de las figuras 6 y 7 , usamos el suavizamento de imagen conocido como rendering, sólo para el valor proporcional a la densidad de las partículas de gas mientras que para las partículas de materia obscura solo se ha colocado en punto azul en sus posiciones. La densidad del gas fue normalizada con la densidad media del volumen de la simulación, y a este cociente de densidades se le calculó el logaritmo de base 10; a este valor se le aplica el rendering. Así pues, cuando la densidad de tales partículas es menor que la densidad media, nos queda un valor negativo en la columna para suavizar; cuando la acumulación de gas es notable, nos queda un valor positivo en dicha columna.

Otra posible pareja de figuras para comparar la misma región se puede construir usando suavizamiento de imagen o rendering usando el valor proporcional a la densidad a todas las partículas disponibles en las simulaciones, tanto de gas como de materia obscura, en la siguiente forma: fijando para éstas últimas el valor arbitrario de cero. Entonces el valor en la columna por suavizar es siempre cero para la materia obscura. El resultado de este procedimiento para las simulaciones de base y modificada se ve en las figuras 8 y 9 , respectivamente.

Vale la pena señalar que las figuras 6, 7, 8 y 9 fueron elaboradas usando el código público de visualización conocido como Paraview [18].

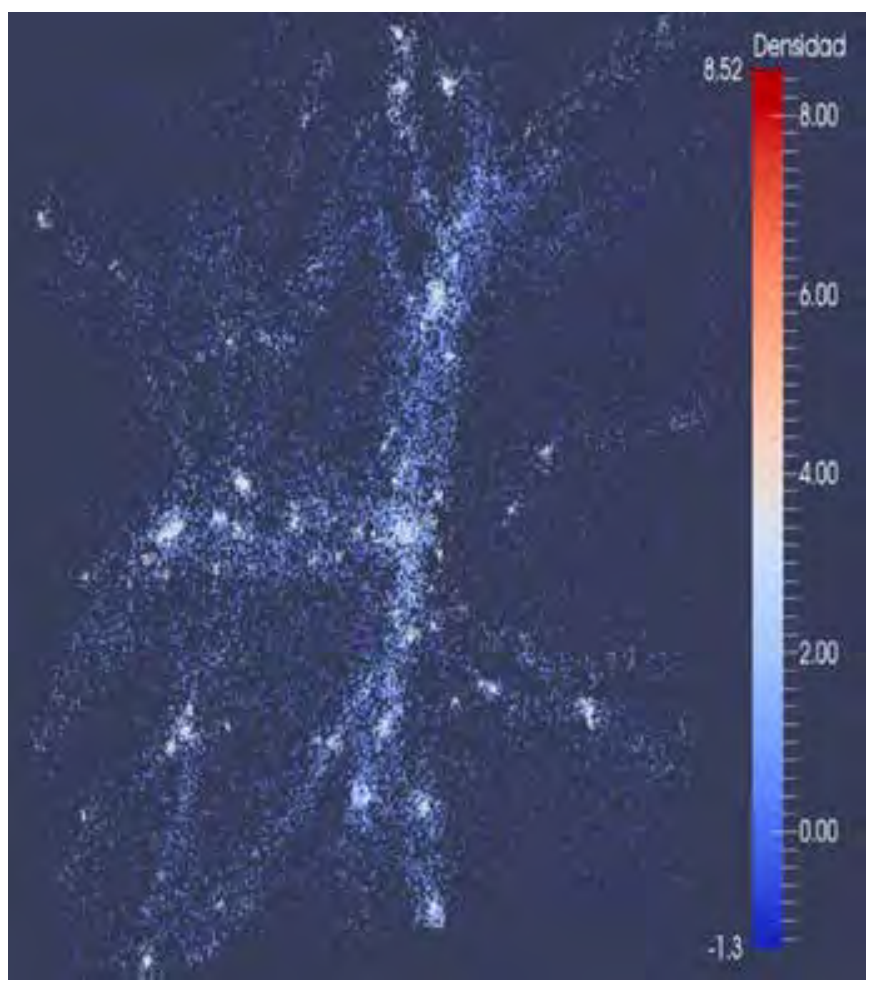

Figura 8. Corte transversal paralelo al plano $X Y$ para mostrar la región del halo mayor encontrado en la simulación base. La escala de colores para la densidad se muestra en la barra vertical.

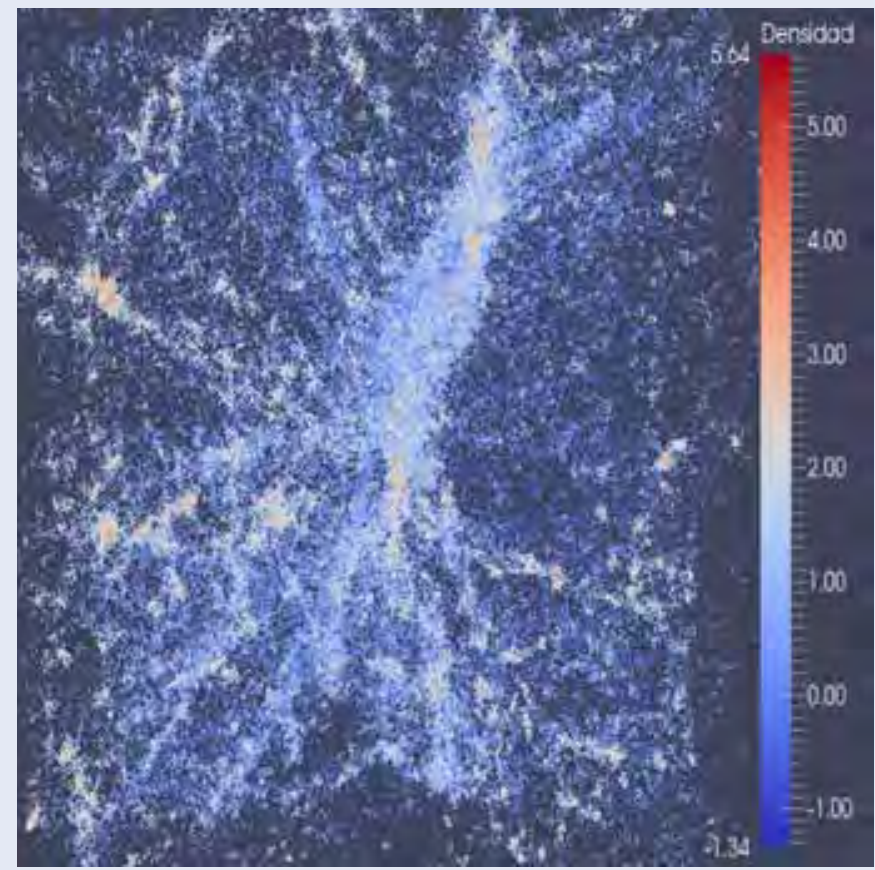

Figura 9. Corte transversal paralelo al plano XY para mostrar la región del halo mayor encontrado en la simulación modificada: en la barra vertical de la derecha se ve la escala de colores para suavizar la densidad.

\section{DISCUSIÓN}

Como la escala de colores se escogió igual en ambas figuras 8 y 9 , es posible establecer una comparación en la distribución de materia, aunque los valores extremos detectados en la columna suavizada, no son iguales en ambas simulaciones, como se puede apreciar en la barra vertical colocada en la parte derecha de cada figura.

Con respecto a la figura 8 , que corresponde a la simulación base, comentamos que en color rojo se ven las partículas menos densas, las que se encuentran dispersas en los alrededores de la región central. La materia obscura se ve en color anaranjado (y colores entre rojo y amarillo, por lo cual notamos que la región central del halo está formado básicamente por materia obscura; también se ven varios halos de materia obscura de menor tamaño en toda la zona circundante al halo central, siendo los halos secundarios más significativos aquellos localizados en la parte izquierda y arriba del halo primario (el central). Las partículas de gas con densidades intermedias crecientes se ven en colores amarillo y verde; se puede notar cierto traslape entre algunos halos menores de materia obscura y estas agregaciones de gas de densidad media. Es necesario hacer un acercamiento (zoom in) en la gráfica para detectar un par de puntos azules, en los cuales la densidad del gas alcanzó el valor máximo detectado en la columna suavizada, el cual se muestra en la parte superior de la barra vertical de colores; y como era de esperarse, tales puntos azules se localizan cerca del núcleo en el halo primario en la región central. 
Con respecto a la figura 9 , aclaramos que el gas adicional en la simulación modificada se ve en color verde; se ve claramente el halo primario de materia obscura y con alguna dificultad, también se pueden observar algunos de los halos de materia obscura secundarios circundantes al central. De hecho, al igual que en la simulación base de la figura 8 , se distingue claramente el brazo grueso que sale del halo central en la dirección noreste. Se puede apreciar la formación de condensaciones bien definidas de este gas (en color verde) a lo largo de múltiples filamentos secundarios conectados al halo principal desde varias direcciones de la zona oeste con respecto de la región central. Estos múltiples filamentos no aparecen todos en la simulación base de la figura 8. En color azul claro se pueden apreciar a simple vista, varias condensaciones de gas con densidades altas; sin embargo, no se ve en donde está localizada la concentración de gas que alcanza la densidad máxima registrada en la barra vertical. Si se trata de un par de puntos aislados como en la simulación de base, entonces es probable que el gas semi-denso en color verde se traslape con la región central hasta ocultar tal concentración.

\section{CONCLUSIÓN}

Se sabe que la formación de estructura en el Universo es un proceso muy complejo de evolución dinámica, en el cual ocurren fenómenos de transporte de masa, momento y energía mediante la interacción hidrodinámica de diferentes fluidos auto-gravitantes.

Como era de esperarse, la distribución de materia obscura observada en la simulación de base, cambia ligeramente en la simulación modificada. Cuantificar estos efectos del gas adicional sobre la simulación es muy importante, ya que es el gas el componente básico que constituye a las galaxias, cuyas propiedades físicas se presentan en los catálogos observacionales, que se han ya construido por ejemplo, WMAP (The Wilkinson Microwave Anisotropy Probe), SDSS (The Sloan Digital Sky Survey) o bien que se están construyendo hoy día, como DES (the Dark Energy Survey) y usando equipo con tecnología de punta, como el SPT (The South Pole Telescope), ACT (Atacama Cosmology Telescope) y LSST (Large Synoptic Survey Telescope).

Conviene destacar que las simulaciones cosmológicas mostradas en este artículo constituyen un primer paso de un proyecto más ambicioso para simular la formación de estructura a nivel cosmológico con gas incluido. En una aproximación posterior a este problema científico, estableceremos una técnica cuantitativa para comparar la distribución del gas adicional en torno al halo de materia obscura, ya que por falta de espacio, no es posible realizar ese análisis en este artículo.

Debemos aclarar que un beneficio adicional de este trabajo es mostrar y comentar acerca de las herramientas libres disponibles hoy día para la realización de simulaciones cosmológicas, puesto que no siempre han estado disponibles los códigos necesarios.

\section{AGRADECIMIENTOS}

El autor desea agradecer al ACARUS de la UNISON, por las facilidades prestadas durante la realización de las simulaciones aquí presentadas.

\section{BIBLIOGRAFÍA}

T. Padmanabhan, "Structure Formation in the Universe", Cambridge University Press, 1998.

[2] P. Coles and F. Lucchin, "Cosmology: the origin and evolution of cosmic structure", Wiley, 2002.

[3]https://en.wikipedia.org/wiki/Cosmic_microwave_ background.

[4] M. Longair, "Galaxy Formation”, Springer-Verlag, 1998.

[5] G.R. Liu, "Smoothed Particle Hydrodynamics", World Scientific Printers, 2003.

[6] The Millennium Simulation Project, 2000, http://wwwmpa. mpa-garching.mpg.de/galform/virgo/millennium/

[7] The Illustris Simulation: Towards a predictive theory of galaxy formation, 2013, http://www.illustris-project.org/.

[8] The Planck Collaboration, "Planck 2013 results. XVI. Cosmological parameters". ArXiv: 1303.5076.

[9] V. Springel, "A code for cosmological simulations of structure formation", Monthly Notices of the Royal Astronomical Society, Vol. 364, pp.1105-1134, 2005. Ver también la liga http://wwwmpa.mpa-garching.mpg.de/ volker/gadget/

[10] J. J. Monaghan and R. A. Gingold, "Shock simulation by the particle method SPH," Journal of Computational Physics, vol. 52, no. 2, pp. 374-389, 1983.

[11] D. S. Balsara, "von Neumann stability analysis of smoothed particle hydrodynamics-suggestions for optimal algorithms,"Journal of Computational Physics, vol. 121, no. 2, pp. 357-372, 1995.

[12] http://www.h-its.org/tap-software-en/ngenic-code/

[13] S. Kitsionas and A.P. Whitworth, "Smooted particle Hydrodynamics with Particle splitting, applied to selfgravitating collapse", Monthly Notices of the Royal Astronomical Society, Volume 330, Issue 1, pp. 129-136,2002.

[14] E. Bertschinger, "SIMULATIONS OF STRUCTURE FORMATION IN THE UNIVERSE", Annual Review of Astronomy and Astrophysics, Vol. 36: 599-654, 1998.

[15] http://guillermoarreagag-difusunison.blogspot.mx

[16] D. Price, Daniel, SPLASH: a free and open source visualisation tool for Smoothed Particle Hydrodynamics (SPH) simulations, 2015. Ver la liga http://users.monash.edu. au/ dprice/splash/.

[17]http://obswww.unige.ch/lastro/misc/TP4/doc/rst/Exercices/ Ex05.html

[18] Paraview, an open-source, multi-platform data analysis and visualization application, http://www.paraview.org/. 